DTPA scan

Resident Corner www.pjkd.com.pk

\title{
Renal DTPA Scan
}

\section{Dr. Ahmad Qureshy \\ DG NM\&O \\ Chief Nuclear Physician \\ Email: aqureshy@yahoo.com}

25 years old male presented with back pain and found to have solitary left kidney on ultrasound.

Left kidney is normal sized, with good perfusion, good cortical uptake well-functioning left kidney without any outflow obstruction and prompt clearance into the urinary bladder.

Right kidney is replaced by a photon deficient area (Red Arrow without any perfusion, tracer cortical uptake or excretion - nonfunctioning kidney

The clearance curves if available would have been more descriptive in terms of uptake and clearance.

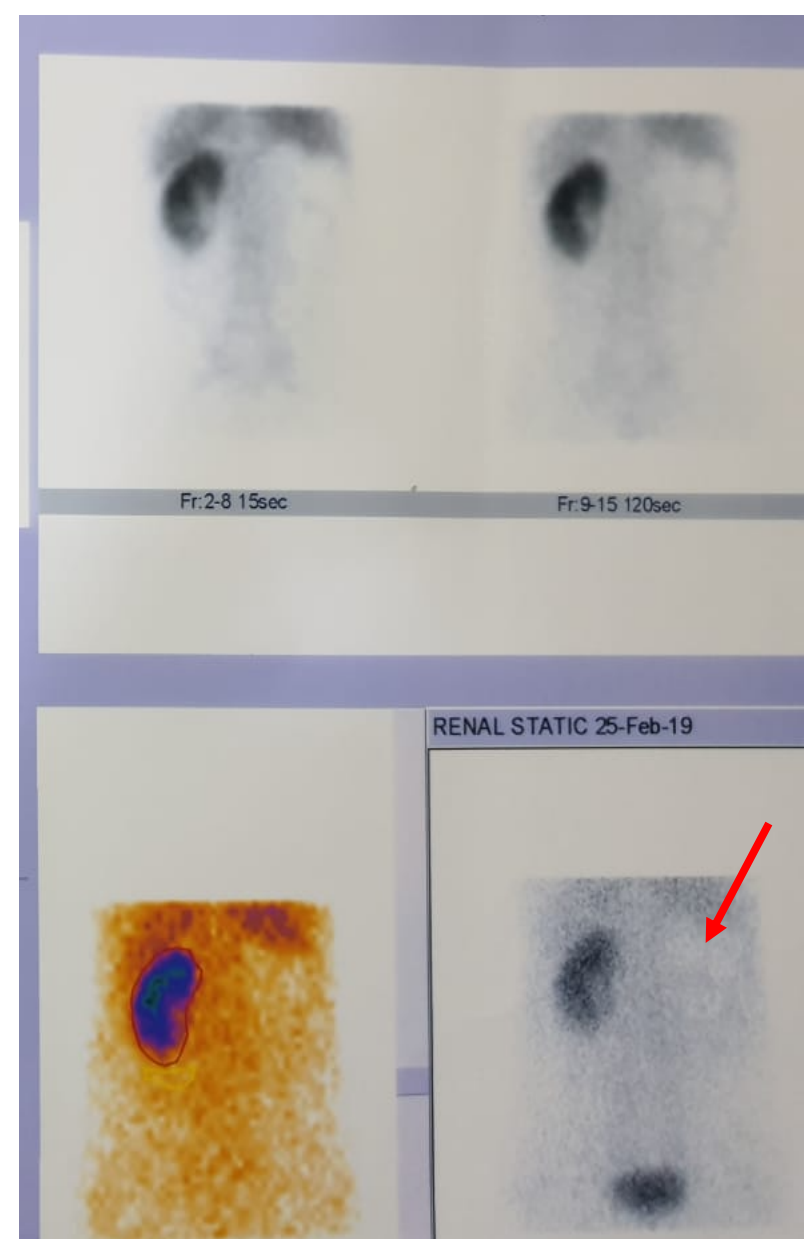

